\title{
Magnetic resonance imaging: the preferred imaging method for evaluation of the right ventricle
}

\author{
L. H. B. Baur
}

Received: 11 May 2008/Accepted: 13 May 2008/Published online: 28 May 2008

(C) The Author(s) 2008

Knowledge of right ventricular size, volume and right ventricular function is important in many cardiac entities such as heart failure, pulmonary disease, congenital heart disease, atrial and ventricular arrhythmias and valvular heart disease. Sequential imaging of right ventricular size and function can be of importance for evaluation of therapy in these diseases. Unfortunately, correct imaging of the right ventricle is difficult due to the complexity of the right ventricular anatomy. This has prevented accurate volume determination by two-dimensional echocardiography. Besides this, echocardiographic imaging is hampered by the location of the right ventricle behind the sternum. Echocardiographic imaging of the right ventricle with $\mathrm{M}$-mode is also subject to variation with $\mathrm{RV}$ position relative to the chest wall and with patient position. Although several geometric assumptions have been made to define the right ventricular geometry, no simple geometric figure accurately defines the right ventricular chamber [1]. Both the area-length method and the Simpson's rule have been undertaken to quantify right ventricular volume and function $[2,3]$. Unfortunately the correlation between the echocardiographic volume and volumes measured with contrast angiography or

L. H. B. Baur ( $ه)$

Department of Cardiology, Atrium Medical Centre

Parkstad, Heerlen, University of Maastricht, Maastricht, Henri Dunantstreet 5, 6410CX Heerlen, The Netherlands e-mail: 1br01@atriummc.nl radionuclide imaging was variable. Although 3D echocardiography is not dependent on geometric assumptions, the correlation with MRI as right ventricular measurements and right ventricular function is only modest and is not better than 2D echocardiographic imaging [4]. The correct paper of Lai et al. [5] showed, that in a group of normal individuals, patients with atrial septal defect and tetralogy of Fallot, there was no difference in the mean study quality between groups for echocardiography and for MRI. However 2D RV parameters were significantly smaller when performed on echocardiography versus MRI. However the interobserver variability for MRI images was significantly lower than the variability for echocardiographic imaging. The correlation between echocardiographic images and MRI images was poor however.

The difference between echocardiography was even larger in those patients in which knowledge of right ventricular volume and function matters: Patients with congential heart disease and RV overload. The results are of importance, because in those patients, in which right ventricular function has to be evaluated, MRI is the technique of choice with a limited intra and inter observer variability and accurate delineation of right ventricular cavity. Although 3D echocardiography does not have the anatomical drawback of 2D echocardiographic imaging, 3D echocardiography faces the same acoustic challenges as 2D echocardiography. In clinical studies $3 \mathrm{D}$ echocardiographic imaging has not proven to 
be superior to magnetic resonance imaging. Larger population studies are needed to prove accuracy of magnetic resonance imaging versus echocardiography and nuclear imaging in different patient populations with right ventricular disease. Until then magnetic resonance imaging, when used correctly and when analysed correctly is the preferred technique to evaluate right ventricular function.

Open Access This article is distributed under the terms of the Creative Commons Attribution Noncommercial License which permits any noncommercial use, distribution, and reproduction in any medium, provided the original author(s) and source are credited.

\section{References}

1. Feigenbaum H, Armstrong WF, Ryan T (eds) (2005) Left atrium, right atrium and right ventricle. In: Feigenbaum's echocardiography, chap 7. Wolters Kluwer, pp 181-213
2. Starling MR, Crawford MH, Sorensen SG, O'Rourke RA (1982) A new two-dimensional echocardiographic technique for evaluating right ventricular size and performance in patients with obstructive lung disease. Circulation 66: 612-620

3. Panidis IP, Ren JF, Kotler MN, Mintz G, Iskandrian A, Ross J, Kane S (1983) Two-dimensional echocardiographic estimation of right ventricular ejection fraction in patients with coronary artery disease. J Am Coll Cardiol 2:911-918

4. Kjaergaard J, Petersen CL, Kjaer A, Schaadt BK, Oh JK, Hassager C (2006) Evaluation of right ventricular volume and function by $2 \mathrm{D}$ and $3 \mathrm{D}$ echocardiography compared to MRI. Eur J Echocardiogr 7(6):430-438

5. Lai et al (2008) Accuracy of guideline recommendations for two-dimensional quantification of the right ventricle echocardiography. Int J Cardiovasc Imaging. doi:10.1007/s10554008-9314-4 